\title{
ПСИХОТЕРАПИЯ $И$
}

\section{МЕДИЦИНСКАЯ ПСИХОЛОГИЯ}

УДК 616.831-001.31:616.831-001.34:616.895-044.332:616.8-085.851:355.292.3

Для цитирования: Стреминский С.Ю., Шереметьева И.И., Строганов А.Е., Лещенко Л.В., Курышкин В.И., Кулешова Е.О. Психотерапевтические симптомы-мишени у комбатантов с органическими заболеваниями головного мозга, сочетанными с расстройством адаптации. Сибирский вестник психиатрии и наркологии. 2020; 2 (107): 67-74. https://doi.org/10.26617/1810-3111-2020-2(107)-67-74

\section{Психотерапевтические симптомы-мишени у комбатантов}

\section{с органическими заболеваниями головного мозга,} сочетанными с расстройством адаптации

\section{1Стреминский С.Ю., 2 Шереметьева И.И., ${ }^{2}$ Строганов А.Е., 2Лещенко Л.В., 2Курышкин В.И., гКулешова Е.О.}

1 «Станичия скорой медицинской помощчи, г. Барнаул»

Россия, 6560496, Барнаул, ул. Ядринцева, 94

${ }^{2}$ Алтайский государственный медицинский университет

Россия, 656038, Барнаул, пр. Ленина, 40

\section{PEЗЮME}

Публикуемые в последние годы данные свидетельствуют об актуальности проблемы коморбидных расстройств в общей структуре психической патологии, что обусловлено её социальной направленностью, первостепенным значением для клинической психиатрии в целом. Цель: определение психотерапевтических симптомов-мишеней, типичных для клинической ситуации, личностной характерологии и нозологической специфики у комбатантов с сочетанием органических заболеваний головного мозга и расстройства адаптации с целью повышения качества оказания лечебно-реабилитационных мероприятий. Проведено аналитическое исследование 102 пациентов неврологического отделения № 3 КГБУЗ «Алтайский краевой госпиталь для ветеранов войн». По результатам исследования определены наиболее характерные для данной группы пациентов социально-демографические и индивидуально-личностные особенности, на основании которых выделены психотерапевтические симптомы-мишени в структуре программ комплексной психосоциореабилитации.

Ключевые слова: сочетанные патологии, психотерапевтические симптомы-мишени, органические заболевания головного мозга, расстройство адаптации, комбатанты.

\section{ВВЕДЕНИЕ}

Второе десятилетие XXI в. отмечено ростом численности больных с сочетанием нескольких хронических заболеваний (коморбидность, мультиморбидность, двойной диагноз, полипатии и др.) Высокий удельный вес смешанных заболеваний отмечается и в общей структуре психической патологии. Подобные заболевания характеризуются полиморфизмом клинической картины из-за их взаимовлияния - высокой вероятностью негативных последствий для жизнедеятельности пациентов [6, 12]. Актуальность проблем сочетанной патологии предопределена ощутимым вкладом коморбидных расстройств в общую структуру психической патологии, её социальным звучанием, значимостью для клинической психиатрии в целом.
Сочетанные формы патологий остаются малоизученной сферой и по-прежнему являются одной из актуальных проблем современной психиатрии [11, 13]. Значимость исследований данного спектра патологий связана также с формированием новых взглядов на общую структуру психической патологии [6, 7, 9].

На сегодняшний день в России более 5 миллионов человек служат в силовых структурах. Большая часть из них длительное время находится в экстремальных условиях профессиональной деятельности. Авторы исследований, посвященных данной проблематике, отмечают, что данный контингент сталкивается с затруднениями в организации медико-социальной помощи и психосоциальной реабилитации. 
К числу таковых препятствий можно отнести несвоевременную диагностику и терапию психических расстройств, ограниченность психопрофилактических, коррекционных мероприятий и психосоциальных мер, обусловливающих успешную интеграцию в гражданское общество [1].

Военнослужащие подвержены воздействию стресса во время службы и по её окончании. При этом по истечении периода острой реакции на стресс в контексте негативной адаптации психическая переработка травматического опыта может не происходить или протекать не в полной мере. В то же время возможно развитие комплекса патологических посттравматических состояний - от нарушения адаптации до её расстройства $[14,15]$. Бывшие военнослужащие нередко подвергаются экзогенным воздействиям. Органические нарушения, как правило, являются причинным фактором возникновения у них когнитивных дисфункций [10].

Наличие неблагоприятных тенденций ухудшения психического здоровья и социальной адаптации участников боевых действий требует разработки системы комплексных мер медицинского характера и социальной защиты, обеспечивающих улучшение здоровья ветеранов боевых действий, а также создание условий для их саморазвития и самореализации, социально значимых компетенций участия в общественной жизни и поступательном движении общества [2]. По мнению В.Я. Семке (2009), к главным задачам современной психиатрической науки относятся необходимость достижения согласия в процессе оказания помощи между терапевтом и пациентом, совершенствование психотерапевтического подхода в работе полипрофессиональной бригады, снижение стигматизации пациента, психиатра и психотерапевта. Определена необходимость интеграции пациента в общество и работу и более широкое использование разных видов терапии (когнитивно-поведенческой, семейной, терапии средой, индивидуально-ориентированной) и децентрализация и оптимизация помощи [8].

На сегодняшний день не существует значительных разногласий среди представителей тех или иных психотерапевтических направлений, касающихся общей структуры психотерапевтического процесса, основных механизмов изменений при психотерапии, а также их стадийности, общих факторов действия психотерапии, переменных со стороны пациента, психотерапевта и взаимодействия между ними [4].
К числу общих представлений в клинических моделях психотерапии относятся такие понятия, как цели, задачи и мишени психотерапии [3].

В методических рекомендациях СанктПетербургского НИ Психоневрологического института им. В.М. Бехтерева предлагается следующая типология «мишеней психотерапии», которая делает возможным системное описание клинических психотерапевтических подходов к лечению обширного диапазона психических расстройств, в отношении которых применима психотерапия:

1. Нозоспецифичные психотерапевтические мишени (психотерапевтические мишени нозологической специфики).

2. Мишени, специфичные для личности пациента.

3. Мишени, специфичные для психотерапевтического процесса.

4. Психотерапевтические мишени, специфичные для клинической ситуации.

5. Мишени, специфичные для психотерапевтического метода.

С нашей точки зрения, данная типология будет актуальной и уместной при сочетанных патологиях у комбатантов. Основанием для этого является то, что она учитывает все возможные аспекты психотерапевтического вмешательства и позволяет соотносить его с широким кругом патологических механизмов, описание которых выходит за пределы теорий отдельных психотерапевтических методов. Данный принцип используемой типологии позволит провести сравнительный анализ эффективности применяемых моделей психотерапии.

\section{ЦЕЛЬ ИССЛЕДОВАНИЯ}

Определение психотерапевтических симптомов-мишеней, характерных для клинической ситуации, особенностей личности и нозологической специфики у комбатантов с сочетанием органических заболеваний головного мозга (ОЗГМ) и расстройства адаптации с целью повышения качества оказания и совершенствования организации комплексных лечебнореабилитационных мероприятий.

\section{МАТЕРИАЛЫ И МЕТОДЫ}

Проведено аналитическое исследование 102 пациентов КГБУЗ «Алтайский краевой госпиталь для ветеранов войн», получавших лечение в период с 2015 г. по 2018 г. Методы: клиникопсихопатологический, клинико-динамический, статистический. Результаты исследования обработаны с помощью пакета STATISTIKA 10.0.228.8 Portable и Microsoft Excel. 
При статистической обработке полученных данных применялись следующие статистические методы: расчет средней арифметической, среднего квадратичного стандартного отклонения для нормального распределения и медианы, нижнего и верхнего квартилей для распределения, отличающегося от нормального; корреляционный анализ по Спирмену. Для оценки статистически значимых различий параметров использовались три уровня значимости: 5\% (вероятность ошибочной оценки равна $\mathrm{p}=0,05), 1 \%$ $(\mathrm{p}=0,01)$. При значении $\mathrm{p}<0,05$ принятую нулевую гипотезу отклоняли на взятом уровне.

\section{РЕЗУЛЬТАТЫ ИССЛЕДОВАНИЯ}

Для определения психотерапевтических симптомов-мишеней был проведен сравнительной анализ 102 пациентов, которые были разделены на 2 группы. К основной группе исследования были отнесены пациенты с ОЗГМ, сочетанными с расстройством адаптации $(\mathrm{n}=45)$. $\mathrm{B}$ группу сравнения $(\mathrm{n}=57)$ включены пациенты с ОЗГМ. Необходимо отметить, что в соответствии с МКБ-10 ОЗГМ были представлены «Неуточненными непсихотическими расстройствами, обусловленными повреждением и дисфункцией головного мозга или соматической болезнью» (F06.92) и расстройством адаптации: «Расстройствами адаптации с преобладанием нарушения других эмоций» (F43.23) - у 77,8\% (35) и «Смешанными тревожными и депрессивными реакциями» (F43.22) - у 22,2\% (10).

Изучение возрастных параметров пациентов обеих групп показало, что возраст пациентов группы сравнения располагался в диапазоне от 28 до 60 лет, медиана возраста и квартильный размах - $48(43 ; 54)$ соответственно. Анализ возрастных особенностей пациентов основной группы отражает, что возраст обследуемых находится в интервале от 30 до 60 лет, медиана возраста и квартильный размах - 49 (44; 53) соответственно, что в целом не отличается от таковых показателей группы сравнения.

Межгрупповая статистическая обработка не выявила достоверных различий по возрастным особенностям среди пациентов сравниваемых групп не только в $\chi^{2}$-процентном соотношении, но и при статистическом анализе с помощью критерия $\chi^{2}(\mathrm{p}>0,05)$. Таким образом, по выявленным возрастным характеристикам группы явились однородно-идентичными.

Изучение особенностей уровня образования исследуемых пациентов при статистическом анализе с помощью критерия $\chi^{2}$ значимых межгрупповых различий не выявило $(\mathrm{p}>0,05)$. Отметим, что пациентов со средним специальным образованием как в основной группе, так и в группе сравнения было больше, чем пациентов с другим образовательным уровнем $(\mathrm{p}<0,05)$. Таким образом, подавляющее большинство исследованных пациентов находились в трудоспособном возрасте и в большинстве случаев получили среднее специальное образование.

При анализе особенностей трудоустройства обнаружено, что безработными в основной группе являлись 40\% (18) пациентов, в группе сравнения - 33,3\% (19); официально трудоустроенными в основной группе оказалось 22,2\% (10) бывших военнослужащих, в группе сравнения таковых было $42,1 \%$ человек (24); неофициально трудоустроенными в основной группе оказалось $37,8 \%$ пациентов (17), в группе сравнения $-24,5 \%$ (14). Таким образом, отмечается низкий процент официально трудоустроенных лиц в основной группе. Сравнение групп с помощью критерия $\chi^{2}$ в свою очередь так же показало, что достоверно меньшее число пациентов основной группы имеют официальное трудоустройство $(\mathrm{p}<0,05)$. Межгрупповые различия трудоустройства пациентов по группам отражены в таблице 1.

Т а б л и ц а 1. Сравнительное распределение по характеру трудоустройства в группах исследования по критерию $\chi^{2}$

\begin{tabular}{|c|c|c|c|c|c|c|}
\hline \multirow{2}{*}{ Группа исследования } & \multicolumn{5}{|c|}{ Профессионально-трудовая занятость пациентов } \\
\cline { 2 - 7 } & \multicolumn{2}{|c|}{ Не работает } & Работает официально & \multicolumn{2}{c|}{ Работает неофициально } \\
\cline { 2 - 7 } & Абс. & $\%$ & Абс. & Абс. & А \\
\hline Основная группа $(\mathrm{n}=45)$ & 18 & 40 & 10 & 22,2 & 17 & 37,8 \\
\hline Группа сравнения $(\mathrm{n}=57)$ & 19 & 33,4 & 24 & 42,1 & 14 & 24,5 \\
\hline p-level & \multicolumn{2}{|c|}{$\mathrm{p}>0,05$} & \multicolumn{2}{c}{$\mathbf{p}<\mathbf{0 5}$} & \multicolumn{2}{c|}{$\mathrm{p}>0,05$} \\
\hline
\end{tabular}

Исследование семейного положения показало, что в основной группе женатых больных оказалось незначительно меньше - 64,4\% (29), чем в группе сравнения - 78,9\% (45). Неблагополучие в семейных отношениях преобладает в основной группе - разведены $28,8 \%$ комбатантов (13) против $12,2 \%$ комбатантов (7) в группе сравнения. Доля холостяков в основной группе $(6,6 \%-3)$ незначительно ниже, чем в группе сравнения $(8,7 \%-5)$. 
Проведенный с помощью критерия $\chi^{2}$ статистический анализ показал, что достоверно большее число представителей основной группы разведены $(\mathrm{p}<0,05)$. Таким образом, в основной группе исследования зарегистрирован более низкий социальный уровень, включая отсутствие официального места трудоустройства, несмотря на трудоспособный возраст (медиана и квартильный размах - 49 (44; 53), а также большая доля расторжения браков.

Все пациенты основной группы и некоторые больные группы сравнения обозначали в качестве факторов риска развития своих невротиче- ских расстройств различные психогенные воздействия, такие как проблемы в семье и с работой, неурядицы в личной жизни, финансовые проблемы и т.д. В группе сравнения психогении были представлены весьма скудно и выявлялись, как правило, только при активном расспросе пациентов, сами исследуемые оценивали их как незначительно интенсивные. С помощью критерия $\chi^{2}$ было установлено, что среди психогенных воздействий самыми распространенными явились проблемы в семье и финансовые сложности $(\mathrm{p}<0,05)$, данные отражены в таблице 2 .

T а б л и ц а 2. Сравнительное распределение психогенных воздействий по причинной обусловленности в группах исследования по критерию $\chi^{2}$

\begin{tabular}{|c|c|c|c|c|}
\hline \multirow[t]{2}{*}{ Причина психогении } & \multicolumn{2}{|c|}{ Группа сравнения $(\mathrm{n}=57)$} & \multicolumn{2}{|c|}{ Основная группа $(\mathrm{n}=45)$} \\
\hline & Абс. & $\%$ & Абс. & $\%$ \\
\hline Проблемы в семье & 2 & 3,5 & $25^{*}$ & 55,5 \\
\hline Проблемы в личной жизни & 1 & 1,7 & $11 *$ & 24,4 \\
\hline Финансовые затруднения & 2 & 3,5 & $20 *$ & 44,4 \\
\hline Проблемы с работой & 2 & 3,5 & $15^{*}$ & 33,3 \\
\hline Судебные разбирательства & 1 & 1,7 & $12 *$ & 26,6 \\
\hline Проблемы со здоровьем родственников & 2 & 3,5 & $12 *$ & 26,6 \\
\hline
\end{tabular}

П р и м е ч а н и е. Достоверность различий: $*-p<0,05$. Разность между количеством пациентов и числом психогений объясняется наличием нескольких причин психогений у одного пациента.

С целью определения психотерапевтических симптомов-мишеней, характерных для личности пациента, было проведено исследование с использованием Личностного опросника Ай- зенка (EPI), по результатам которого был выполнен межгрупповой анализ. Полученные данные после систематизации были проанализированы (табл. 3).

Т а б л и ц а 3. Сравнительное распределение результатов согласно тесту Айзенка (EPI)в группах исследования по критерию U Манна-Уитни

\begin{tabular}{|c|c|c|c|c|c|c|}
\hline \multicolumn{3}{|c|}{ Группа сравнения $(\mathrm{n}=57)$} & \multirow[t]{2}{*}{ p-level } & \multicolumn{3}{|c|}{ Основная группа (n=45) } \\
\hline Шкала & Сумма рангов & $\mathrm{M}$ & & $\mathrm{M}$ & Сумма рангов & Шкала \\
\hline Интроверсия & 2537,5 & 1,8 & $0,00\left(\mathrm{U}_{\text {эмп }}=884\right)$ & 4,2 & 2715,5 & Интроверсия \\
\hline Экстраверсия & 3244,5 & 6,6 & $0,03\left(\mathrm{U}_{\text {эмп }}=973\right)$ & 3,2 & 2008,5 & Экстраверсия \\
\hline Нейротизм & 1653 & 0,3 & $0,00\left(\mathrm{U}_{\text {эмп }}=0,0\right)$ & 13,4 & 3600 & Нейротизм \\
\hline
\end{tabular}

По итогам сравнительного распределения результатов выяснилось, что уровни интроверсии и нейротизма в основной группе оказались более выраженными $(\mathrm{p}<0,05)$. Это совпадает с данными о том, что интроверты характеризуются крайней степенью самоорганизации и педантичности, высоким уровнем тревожности и эмоциональной неустойчивости в случаях нейротизма, что, как следствие, приводит к невротизации таких личностей.

Для выделения психотерапевтических симптомов-мишеней клинико-нозологической специфики нами была разработана схема определения особенностей распространенности и выраженности нозоспецифических психотерапевтических симптомов-мишеней.
Выраженность нозоспецифических симптомов оценивалась нами по 4-балльной шкале, где: 0 баллов - отсутствие симптома, 1 балл незначительная степень, 2 балла - умеренная степень, 3 балла - выраженные симптомы. Под незначительно выраженными симптомами подразумевались кратковременные, однократно или эпизодически проявляющиеся расстройства, не вызывающие беспокойства, купирующиеся без приема медицинских препаратов. Под умеренно выраженными - симптомы, повторяющиеся время от времени, обращающие на себя внимание, купирующиеся приемом медицинских препаратов. Выраженные симптомы отличались протяженностью во времени и не купировались без специализированной медицинской помощи. 
Исследование проводилось поэтапно. На первом этапе были определены наиболее распространенные симптомы в изучаемых группах исследования с помощью критерия $\chi^{2}$. Второй этап был предназначен для определения степени выраженности наиболее распространенных симптомов с помощью U-критерия Манна-Уитни.
Данное исследование показало, что наиболее распространенными и выраженными симптомами сочетанных патологий являются раздражительность, утомляемость, эмоциональная неустойчивость, дисфория, пониженное настроение, неосвежающий сон, а также отсутствие чувства сна $(\mathrm{p}<0,05)$. Полученные результаты представлены в таблице 4.

Т а б л и ц а 4. Сравнительное распределение выраженности клинических проявлений заболевания в группах исследования по критерию U Манна-Уитни

\begin{tabular}{|l|c|c|}
\hline \multicolumn{1}{c}{ Особенность } & \multicolumn{2}{c|}{ Выраженность нозоспецифических симптомов-мишеней (медиана) } \\
\cline { 2 - 3 } \multicolumn{1}{c|}{ клинических проявлений } & Группа сравнения $(\mathrm{n}=57)$ & Основная группа $(\mathrm{n}=45)$ \\
\hline Повышенная утомляемость & $1(0 ; 1)$ & $1(1 ; 2)^{*}$ \\
\hline Раздражительность & $1(1 ; 2)$ & $2(2 ; 2)^{*}$ \\
\hline Эмоциональная неустойчивость & $1(0 ; 1)$ & $1(1 ; 2)^{*}$ \\
\hline Дисфория & $0(0 ; 1)$ & $3(2 ; 3)^{*}$ \\
\hline Пониженное настроение & $0(0 ; 0)$ & $3(2 ; 3)^{*}$ \\
\hline Неосвежающий сон & $0(0 ; 1)$ & $1(1 ; 2)^{*}$ \\
\hline Отсутствие чувство сна & $0(0 ; 0)$ & $1(1 ; 2)^{*}$ \\
\hline
\end{tabular}

П р и м е ч а н и е. Достоверность различий: * - p<0,05. Представленные симптомы являются специфичными для сочетанных форм патологий.

\section{ОБСУЖДЕНИЕ}

Исходя из выявленных нами социальнодемографических особенностей, этиологической факторности, индивидуально-личностных характеристик, а также с учетом схемы определения особенностей распространенности и выраженности нозоспецифических психотерапев- тических симптомов-мишеней, были выделены психотерапевтические симптомы-мишени, характерные для комбатантов с сочетанными (ОЗГМ + расстройство адаптации) патологиями, которые более детально представлены в таблице 5.

\section{Т а б л и ц а 5. Психотерапевтические симптомы-мишени, характерные для комбатантов} с сочетанием органических заболеваний головного мозга и расстройства адаптации

\begin{tabular}{|c|c|c|}
\hline $\begin{array}{c}\text { Название } \\
\text { группы мишеней }\end{array}$ & $\begin{array}{c}\text { Сфера определения } \\
\text { мишеней психотерапии }\end{array}$ & $\begin{array}{c}\text { Содержание мишеней } \\
\text { для проведения психотерапии }\end{array}$ \\
\hline $\begin{array}{c}\text { Симптомы-мишени, } \\
\text { специфичные для } \\
\text { клинической ситуации }\end{array}$ & $\begin{array}{l}\text { Условия жизни и микросоциаль- } \\
\text { ного функционирования пациента, } \\
\text { в первую очередь оказывающие } \\
\text { психогенное и патопластическое } \\
\text { влияние на формирование и раз- } \\
\text { витие расстройства }\end{array}$ & $\begin{array}{l}\text { Отсутствие официального места трудоустрой- } \\
\text { ства, несмотря на трудоспособный возраст (ме- } \\
\text { диана и квартильный размах - } 49 \text { (44; 53). } \\
\text { Семейное неблагополучие, выражающееся в } \\
\text { большем числе разводов в основной группе. } \\
\text { Психогении, связанные с разными жизненными } \\
\text { ситуациями, самые распространенные из них } \\
\text { проблемные ситуации в семье и финансовые } \\
\text { сложности (отсутствие стабильного дохода) }\end{array}$ \\
\hline $\begin{array}{c}\text { Симптомы-мишени, } \\
\text { специфичные для лич- } \\
\text { ностной характерологии }\end{array}$ & $\begin{array}{l}\text { Психологические характеристики } \\
\text { пациентов, существенно влияю- } \\
\text { щие на возникновение и клиниче- } \\
\text { скую динамику расстройства }\end{array}$ & $\begin{array}{l}\text { Нейротизм. } \\
\text { Интроверсия }\end{array}$ \\
\hline $\begin{array}{l}\text { Нозоспецифические } \\
\text { симптомы-мишени }\end{array}$ & $\begin{array}{l}\text { Феномены, описываемые языком } \\
\text { клинической семиотики и специ- } \\
\text { фичные для заболевания }\end{array}$ & $\begin{array}{l}\text { Раздражительность }(\mathrm{p}<0,05) . \\
\text { Утомляемость }(\mathrm{p}<0,05) \text {. } \\
\text { Эмоциональная неустойчивость }(\mathrm{p}<0,05) \text {. } \\
\text { Дисфория }(\mathrm{p}<0,05) \text {. } \\
\text { Пониженное настроение }(\mathrm{p}<0,05) \text {. } \\
\text { Неосвежающий сон }(\mathrm{p}<0,05) . \\
\text { Отсутствие чувства сна }(\mathrm{p}<0,05)\end{array}$ \\
\hline
\end{tabular}


С нашей точки зрения, выявленные в исследовании психотерапевтические симптомымишени могут явиться базовым фундаментом для проведения клинически ориентированной психотерапии, способствовать профессиональной коммуникации между психотерапевтами и другими участниками психотерапевтического процесса, четко и корректно разделить сферы специальной, социальной и индивидуальной компетенций в лечебном процессе, а также повысить эффективность проводимых психореабилитационных мероприятий для пациентов с сочетанными формами патологий.

\section{ВЫВОДЫ}

По результатам собственного исследования определены три группы основных психотерапевтических симптомов-мишеней, характерных для клинической ситуации в основной группе пациентов: 1) отсутствие официального места трудоустройства, несмотря на трудоспособный возраст, медиана и квартильный размах - 49 $(44 ; 53)$; 2) семейное неблагополучие, выражающееся в большем количестве расторгаемых браков (разводы) $(\mathrm{p}<0,05) ; 3)$ психогении, связанные с различными жизненными ситуациями, самыми распространенными из числа которых явились проблемы в семье и финансовые сложности из-за нестабильного дохода $(\mathrm{p}<0,05)$.

Исследованы и обозначены психотерапевтические симптомы-мишени, характерные для личностного профиля пациентов: интроверсия $(\mathrm{p}<0,05)$ и нейротизм $(\mathrm{p}<0,05)$.

Выделены психотерапевтические симптомымишени в соответствии со спецификой нозологических категорий: раздражительность $(\mathrm{p}<0,05)$, утомляемость $(\mathrm{p}<0,05)$, эмоциональная неустойчивость $(\mathrm{p}<0,05)$, дисфория $(\mathrm{p}<0,05)$, пониженное настроение $(\mathrm{p}<0,05)$, неосвежающий сон $(\mathrm{p}<0,05)$, отсутствие чувства сна $(\mathrm{p}<0,05)$.

\section{КОНФЛИКТ ИНТЕРЕСОВ}

Авторы декларируют об отсутствии явных и потенциальных конфликтов интересов в связи с публикацией данной статьи.

\section{ИСТОЧНИК ФИНАНСИРОВАНИЯ}

Авторы заявляют об отсутствии финансирования или спонсорской помощи при проведении исследования.

\section{СООТВЕТСТВИЕ ПРИНЦИПАМ ЭТИКИ}

Исследования были одобрены локальным этическим комитетом при ФГБОУ ВО «Алтайский государственный медицинский университет» Минздрава России (протокол № 02 от 26.02.2020 г.).

\section{ЛИТЕРАТУРА/REFERENCES}

1. Соловьев А.Г., Шутова А.А., Злоказова М.В., Ичитовкина Е.Г. Динамика формирования психических расстройств у комбатантовпенсионеров Министерства внутренних дел. Успехи геронтологии. 2017; 30(6): 912-916. Soloviev A.G., Shutova A.A., Zlokazova M.V., Ichitovkina E.G. The dynamics of the formation of mental disorders in combatants-pensioners of the Ministry of the Interior. Uspekhi gerontologii - the Success of Gerontology. 2017; 30 (6): 912-916 (in Russian).

2. Ичитовкина Е.Г., Соловьев А.Г. Факторы и резервы улучшения здоровья ветеранов боевых действий. Национальные демографические приоритеты: новые подходы, тенденции: сб. науч. тр. Серия: Демография. Социология. Экономика / под ред. С.В. Рязанцева, Т.К. Ростовской. М., 2019: 65-68. Ichitovkina E.G., Soloviev A.G. Factors and reserves for improving the health of war veterans. National demographic priorities: new approaches, trends: collection of scientific papers. Series: Demography. Sociology. Economy. Edited by S.V. Ryazantsev, T.K. Rostovskaya. Moscow, 2019: 65-68 (in Russian).

3. Карвасарский Б.Д. Психотерапия: учебник для вузов под общ. ред. Б.Д. Карвасарского. 3-е изд. СПб., 2008: 672. Karvasarskiy B.D. Psychotherapy: a textbook for universities under the general editorship of B.D. Karvasarskiy. 3rd edition. St. Petersburg, 2008: 672 (in Russian).

4. Пере М., Бауман У. Клиническая психология. СПб., 2012: 943. Pere M., Bauman W. Clinical Psychology. St. Petersburg, 2012: 943 (in Russian).

5. Бохан Н.А., Стоянова И.Я., Счастный Е.Д., Королев А.А. Патопсихологические характеристики пациентов с двойным диагнозом в контексте суицидального поведения. Суицидология. 2014; 5(2): 55-59. Bokhan N.A., Stoyanova I.Ya., Schastnyy E.D., Korolev A.A. Pathopsychological characteristics of patients with a double diagnosis in the context of suicidal behavior. Suitsidologiya Suicidology. 2014; 5(2): 55-59 (in Russian).

6. Пивень Б.Н., Булат О.С., Лещенко Л.В. К диагностике сочетанных форм психических заболеваний. Независимый психиатрический журнал. 2012; 2: 14-15. Piven B.N., Bulat O.S., Leshchenko L.V. To the diagnosis of combined forms of mental illness. Nezavisimyy psikhiatricheskiy zhurnal - Independent Psychiatric Journal. 2012; 2: 14-15 (in Russian).

7. Семке А.В., Мальцева Ю.Л. Шизофрения, сочетанная с соматической патологией: клинические, адаптационные и реабилитационные аспекты. Сибирский вестник психиатрии и наркологии. 2009; 3(54): 42-44. Semke A.V., Maltseva Yu.L. Schizophrenia, combined with somatic pathology: clinical, adaptive and rehabilitative aspects. Sibirskiy vestnik psikhiatrii $i$ narkologii Siberian Herald of Psychiatry and Addiction Psychiatry. 2009; 3(54): 42-44 (in Russian). 
8. Семке В.Я., Рудницкий В.А., Епанчинцева Е.M., Ошаев С.А., Ветлугина Т.П., Никитина В.Б. Сравнительная характеристика влияния интенсивных психотравм различной природы (на примере экологической катастрофы и боевого стресса). Сибирский вестник психиатрии и наркологии. 2009; 6(57): 89-93. Semke V.Ya., Rudnitsky V.A., Epanchintseva E.M., Oshayev S.A., Vetlugina T.P., Nikitina V.B. Comparative characteristic of influence of intense psychotraumata of various natures (on example of ecological catastrophe and combat stress. Sibirskiy vestnik psikhiatrii i narkologii - Siberian Herald of Psychiatry and Addiction Psychiatry. 2009; 6(57): 8993 (in Russian).

9. Шереметьева И.И. Смешанные формы психических заболеваний у диспансерного контингента больных (клинико-статистическое исследование) : автореф. дис. ... канд. мед. наук. Новосибирск, 2001: 21. Sheremetyeva I.I. Mixed forms of mental illness in the dispensary contingent of patients (clinical and statistical study): $\mathrm{PhD}$ thesis. Novosibirsk, 2001: 21 (in Russian).

10. Shetty A.K., Mishra V., Kodali M., Hattiangady B. Corrigendum: Blood brain barrier dysfunction and delayed neurological deficits in mild traumatic brain injury induced by blast shock waves. Front Cell Neurosci. 2014 November; 13(8): 232. DOI: 10.3389/fncel.2014.00404

11. McLean G., Hindle J.V., Guthrie B., Mercer S.W. Comorbidity and polypharmacy in Parkinson's dis- ease: insights from a large Scottish primary care database. BMC Neurol. 2017 Jul 1;17(1):126. http://dx.doi.org/10.1186/s12883-017-0904-4

12. Cancino A., Leiva-Bianchi M., Serrano C. Ballesteros-Teuber S., Cáceres C., Vitriol V. Factors Associated with Psychiatric Comorbidity in Depression Patients in Primary Health Care in Chile. Depression Research and Treatment. 2018 October; 4:1-9. DOI: $10.1155 / 2018 / 1701978$

13. Mukeshimana M., Mchunu G. Management of CoMorbidity of Depression and Chronic NonCommunicable Diseases in Rwanda. Ethiop $J$ Health Sci. 2017 Jan; 27(1): 17-26. DOI: $10.4314 /$ ejhs.v27i1.4

14. Williams J.L., McDevitt-Murphy M.E., Murphy J.G., Crouse E.M. Postconcussive Symptoms, PTSD, and Medical Disease Burden in TreatmentSeeking OEF/OIF/OND Veterans. Military Medicine. $2017 \quad$ March; 182(3):e1645-e1650. DOI: 10.7205/MILMED-D-16-00221

15. Schubert C.F., Schmidt U., Rosner R. Posttraumatic growth in populations with posttraumatic stress disorder a systematic review on growth-related psychological constructs and biological variables. Clin Psychol Psychother. 2016 Nov;23(6):469-486. DOI: $10.1002 / \mathrm{cpp} .1985$

Поступила в редакцию 27.01.2020 Утверждена к печати 01.06.2020

Стреминский Сергей Юрьевич, заместитель главного врача по оперативной работе КГБУЗ «ССМП, г.Барнаул». ORCID ID 0000-0003-1043-3312.

Шереметьева Ирина Игоревна, д.м.н., профессор, и.о. ректора ДПО ФГБОУ ВО «Алтайский государственный медицинский университет» Минздрава России. ORCID ID 0000-0001-8513-9869. sheremeteva-ira@mail.ru

Строганов Александр Евгеньевич, д.м.н., профессор кафедры психиатрии, медицинской психологии и неврологии с курсом ДПО ФГБОУ ВО «Алтайский государственный медицинский университет» Минздрава России. ORCID ID 0000-0002-1791-7996. jazz200261@ mail.ru

Лещенко Лариса Владимировна, к.м.н., доцент кафедры психиатрии, медицинской психологии и неврологии с курсом ДПО ФГБОУ ВО «Алтайский государственный медицинский университет» Минздрава России. ORCID ID 0000-0003-4819-5575. leschenkolvmedik@ mail.ru

Курышкин Владимир Иванович, к.м.н., доцент кафедры психиатрии, медицинской психологии и неврологии с курсом ДПО ФГБОУ ВО «Алтайский государственный медицинский университет» Минздрава России. ORCID ID 0000-0002-2248-9850. kuryshkinv@ mail.ru

Кулешова Елена Олеговна, ассистент кафедры психиатрии, медицинской психологии и неврологии с курсом ДПО ФГБОУ ВО «Алтайский государственный медицинский университет» Минздрава России. ORCID ID 0000-0002-4122-8138. emoryll@ rambler.ru

Стреминский Сергей Юрьевич, stremindoktor@mail.ru 
For citation: Streminsky S.Yu., Sheremetyeva I.I., Stroganov A.E., Leshchenko L.V., Kuryshkin V.I., Kuleshova E.O. Psychotherapeutic target symptoms in combatants with organic brain diseases associated with an adaptation disorder. Siberian Herald of Psychiatry and Addiction Psychiatry. 2020; 2 (107): 67-74. https://doi.org/10.26617/18103111-2020-2(107)-67-74

\title{
Psychotherapeutic target symptoms in combatants with or- ganic brain diseases associated with adjustment disorder
}

\section{Streminsky S.Yu., 2Sheremetyeva I.I., 2Stroganov A.E., 2Leshchenko L.V., 2Kuryshkin V.I., 2Kuleshova E.O.}

\author{
${ }^{1}$ Regional Public Health Institution "Ambulance Station, city of Barnaul” \\ Yadrintsevskaya Street 9, 6560496, Barnaul, Russian Federation \\ ${ }^{2}$ Altai State Medical University of the Ministry of Health and Social Development of Russia \\ Lenin Avenue 40, 656038, Barnaul, Russian Federation
}

\section{ABSTRACT}

The data published in recent years indicate the urgency of the problem of comorbid disorders in the structure of mental pathology, which is due to its social orientation, which is of primary importance for clinical psychiatry as a whole. Objective: determination of target-oriented psychotherapeutic symptoms typical of the clinical situation, personal characterology and nosological specificity of combatants with a combination of organic brain diseases and adjustment disorder in order to improve the quality of therapeutic and rehabilitation measures. An analytical study of 102 patients of the neurological department no. 3 of the "Altai Hospital for Veterans" of Barnaul KSBIH has been conducted. According to the results of the study, the most typical socio-demographic and individual-personality characteristics for this group of patients have been identified, based on which psychotherapeutic symptoms-targets have been identified in the structure of complex psychosocial rehabilitation programs.
\end{abstract}

Keywords: combined pathologies, target psychotherapeutic symptoms, organic brain diseases, adjustment disorderder, combatants.

Received January 27.2020

Accepted June 01.2020

Streminsky Sergey Yu., Deputy Chief Physician for Operational Work, Regional Public Health Institution “Ambulance Station, city of Barnaul”. ORCID ID 0000-0003-1043-3312.

Sheremetyeva Irina I., MD, Professor, Acting Rector, Altai State Medical University of the Ministry of Health and Social Development of Russia. ORCID ID 0000-0001-8513-9869. sheremeteva-ira@mail.ru

Stroganov Alexander E., MD, Professor of the Department of Psychiatry, Medical Psychology and Neurology with a course of additional professional education, Altai State Medical University of the Ministry of Health and Social Development of Russia. ORCID ID 0000-0002-1791-7996. jazz200261@ mail.ru

Leshchenko Larisa V., PhD, Associate Professor of the Department of Psychiatry, Medical Psychology and Neurology with a course of additional professional education, Altai State Medical University of the Ministry of Health and Social Development of Russia. ORCID ID 0000-0003-4819-5575. leschenkolvmedik@ mail.ru

Kuryshkin Vladimir I., PhD, Associate Professor of the Department of Psychiatry, Medical Psychology and Neurology with a course of additional professional education, Altai State Medical University of the Ministry of Health and Social Development of Russia. ORCID ID 0000-0002-2248-9850. kuryshkinv@mail.ru

Kuleshova Elena Olegovna, Assistant of the Department of Psychiatry, Medical Psychology and Neurology with a course of additional professional education, Altai State Medical University of the Ministry of Health and Social Development of Russia. ORCID ID 0000-0002-4122-8138. emoryll@rambler.ru

\section{Streminsky Sergey Yu., stremindoktor@mail.ru}

